\title{
ORTHOMODULARITY AND THE DIRECT SUM OF DIVISION SUBRINGS OF THE QUATERNIONS
}

\author{
RONALD P. MORASH
}

\begin{abstract}
Let $D$ be any division subring of the real quaternions $H$. Let $\oplus D$ denote the linear space of all finitely nonzero sequences from $D$ and let $L$ denote the lattice of all " $\perp$-closed" subspaces of $\oplus D$, where " $\perp$ " denotes the orthogonality relation derived from the $H$-valued form $(a, b)=\sum\left(a_{i} b_{i}^{*}: i=1,2, \cdots\right)$ where $a, b \in \oplus D$, $a=\left(a_{1}, a_{2}, \cdots, a_{N}, 0,0, \cdots\right)$ and $b=\left(b_{1}, b_{2}, \cdots, b_{M}, 0,0, \cdots\right)$, and $b_{i}^{*}$ is the quaternionic conjugate of $b_{i}$. Then, the lattice $L$ is complete and orthocomplemented, but is not orthomodular.
\end{abstract}

1. Introduction. The lattice of all closed subspaces of separable complex Hilbert space is complete, orthocomplemented, atomistic, irreducible, separable, $M$-symmetric, and orthomodular [8]. Let us call a lattice having these seven properties a Hilbert lattice. The dimension of a Hilbert lattice is the cardinality of any maximal family of orthogonal atoms. We have three examples of nonorthoisomorphic infinite-dimensional Hilbert lattices, namely those built on real, complex, and quaternionic Hilbert space. The principal motivation for the investigations of this paper and also of our paper ([8], [9]) is our desire to answer the question: Are there any other infinite-dimensional Hilbert lattices?

In ([8], [9]) we constructed the lattice $L=L\left(l_{2}(D)\right)$ of all " $\perp$-closed" subspaces of the space $l_{2}(D)$ of square-summable $D$ sequences, $D$ an arbitrary division subring of the quaternions, and showed that $L$ is orthomodular if and only if $D$ is the reals, the complex numbers, or the quaternions. (We amplify some of the details of the proof of that theorem in $\S 2$ of this paper.) This result eliminated a class of possible new examples of infinite-dimensional Hilbert lattices and lent credence to the possibility that the answer to our main question is "no". In this paper, we consider the direct sum $\oplus D$ rather than $l_{2}(D)$, and show that its lattice of " $\perp$ closed" subspaces is never orthomodular, for any $D$. This eliminates another class of possible new examples of infinite-dimensional Hilbert lattices, and is additional evidence in support of the possibility that the answer to our main question is "no". Some special cases of this result

Presented to the Society January 19, 1972; received by the editors December 2, 1971 and, in revised form, February 28, 1972.

AMS 1969 subject classifications. Primary 0640; Secondary 4615.

Key words and phrases. Orthomodular lattices.

(c) American Mathematical Society 1972 
follow from already-known facts. If $D$ is closed under quaternionic conjugation, the nonorthomodularity of $L(\oplus D)$ can be concluded from a fact stated by Kaplansky, in a 1950 paper [5, p. 4, bottom paragraph]. Even more specifically, if $D$ is metrically complete, the conclusion of our theorem follows from the theorem of Amemiya and Araki. Our original proof used some key ideas from their paper [1]. We do not give this proof here, but rather proceed, in $\S 3$, by indicating a proof of Kaplansky's result (a proof which uses ideas of Fischer and Gross [3]), and showing how this proof can be used to derive the direct sum result, for $D$ any division subring of the quaternions, in a manner much simpler than our original proof.

The author wishes to thank Professors H. R. Fischer, D. J. Foulis, D. R. Hayes, and I. Kaplansky for suggestions which contribute to the writing of this paper. He especially wishes to acknowledge the help of Professor S. S. Holland, Jr., who directed the author's doctoral dissertation, from which the results of this paper are drawn.

2. $l_{2}(D)$ and its " $\perp$-closed" subspaces. A surprising fact, not explicitly pointed out in ([8], [9]), is that there exist vectors $a \in l_{2}(D)$ such that the one-dimensional subspace $D a$ is not " $\perp$-closed", that is, $D a \subsetneq(D a)^{\perp \perp}$. For example, let $D$ be a division subring of the quaternions which fails to be closed under quaternionic conjugation. In $l_{2}(D)$, for $i=1,2, \cdots$, let $e_{i}$ be the vector having 1 in the $i$ th component and 0 in every other. Choose $x \in D$ such that $x^{*} \notin D, x^{*}$ being the quaternionic conjugate of $x$. Letting $a=(1, x, 0,0, \cdots)$, we note that $e_{1} \in(D a)^{\perp \perp}$, in fact, $(D a)^{\perp \perp}=$ $D e_{1}+D e_{2}$. However, it is true that, for those vectors

$$
a=\sum\left(a_{i} e_{i}: i=1,2, \cdots\right)
$$

which have $a_{i}^{*} \in D$ for all $i=1,2, \cdots$, we have $(D a)^{\perp \perp}=D a$. In fact, we can make the following stronger statement:

2.1. Lemma. Let $r, s$ be nonzero vectors in $l_{2}(D)$,

$$
r=\sum\left(r_{i} e_{i}: i=1,2, \cdots\right) \text { and } s=\sum\left(s_{i} e_{i}: i=1,2, \cdots\right) \text {, }
$$

where $r_{i}, s_{i}, r_{i}^{*}$, and $s_{i}^{*}$ are elements of $D$ for all $i=1,2, \cdots$. Then,

(i) Dr is a closed subspace of $l_{2}(D)$, as is $D s$,

(ii) $D r+D s$ is a closed subspace of $l_{2}(D)$.

Proof. (i) Let $t \in(D r)^{\perp \perp}$, where $t=\sum\left(t_{i} e_{i}: i=1,2, \cdots\right)$. It is necessary to show that $t \in D r$, that is, there exists $d \in D$ such that $t_{i}=d r_{i}$, for all $i=2,3, \cdots$. Normalizing, we may assume that $r_{1}=1$. Now, for each $i=2,3, \cdots$, let $v_{i}$ be the vector having $-r_{i}^{*}$ in the first component, 1 in the $i$ th component, and zeros elsewhere. Clearly, each $v_{i}$ is orthogonal to 
$r$ and so, by our initial assumption, to $t$. Thus, for $i=2,3, \cdots$, we get $-t_{1} r_{i}+t_{i}=0$, or $t_{i}=t_{1} r_{i}$. Hence $t=t_{1} r \in D r$.

(ii) Let $t \in(D r+D s)^{\perp \perp}$, where $t=\sum\left(t_{i} e_{i}: i=1,2, \cdots\right)$. Again, normalize to get $r_{1}=1, r_{2}=0, s_{1}=0, s_{2}=1$ (this being valid since we can assume $r$ and $s$ to be linearly independent). Now, for $i=3,4, \cdots$, let $w_{i}$ be the vector $-r_{i}^{*}$ in the first component, $-s_{i}^{*}$ in the second component, 1 in the $i$ th component, and zeros elsewhere. Clearly, each $w_{i} \in(D r+D s)^{1}$, so that $t$ is orthogonal to $w_{i}$ for all $i \geqq 3$. But this means that $t_{i}=t_{1} r_{i}+$ $t_{2} s_{i}$, for $i=3,4, \cdots$, so that $t=t_{1} r+t_{2} s$, as was claimed.

2.2. Corollary. If $r, s \in l_{2}(D), r=\sum\left(r_{i} e_{i}: i=1,2, \cdots\right)$ and $s=$ $\sum\left(s_{i} e_{i}: i=1,2, \cdots\right)$, where $r_{i}$ and $s_{i}$ are all rational numbers, then

(i) $D r$ and $D s$ are closed subspaces of $I_{2}(D)$,

(ii) $D r+D$ s is a closed subspace of $l_{2}(D)$.

Corollary 2.2 provides detailed justification for the construction of the atoms $a=D x, b=D y$ in the proof of the main theorem of [8]. (In that proof, we wrote $a=\operatorname{sp}(x), b=\operatorname{sp}(y)$ for $a=D x, b=D y$, respectively.) Since the vectors $x, y$ used there are rational, Corollary 2.2 asserts that $a, b$ are atoms in $L$ and that $a \vee b=D x+D y$. Then, by the orthomodularity (or the $M$-symmetry), we must have $(a \vee b) \wedge a^{\perp} \neq 0$, which leads, as we show in [8], to the fact that $\gamma \in D$, for every real $\gamma$. Hence, $R \subseteq D$, and then one concludes easily that either $D=R, D=H$, or $D$ is isometrically isomorphic to the complex numbers.

In [9], we pointed out that our proof of the atomicity and irreducibility of $L$ contained an oversight-the difficulty lying in the fact, pointed out above, that unless $D$ is conjugate-closed, there are vectors $a \in l_{2}(D)$ such that $(D a) \perp \not D D$. In spite of some effort, we have not been able to settle this question, and we therefore do not know whether $L$ is atomistic and irreducible in general.

3. $\oplus D$. In [1], I. Amemiya and H. Araki prove that the lattice $L$ of all closed subspaces of a real or complex inner product space is orthomodular if and only if the inner product space is complete, that is, a Hilbert space. A consequence of this theorem is that, in the linear space of all finitely nonzero sequences of real or complex numbers, the lattice of all $\perp$-closed subspaces is nonorthomodular, where " $\perp$ " is the orthogonality relation generated by the form $(a, b)=\sum\left(a_{i} b_{i}^{*}: i=1, \cdots, N\right)$, where $a=a_{1} e_{1}+\cdots+a_{Y} e_{X}$ and $b=b_{1} e_{1}+\cdots+b_{M I} e_{M I}$, the $a_{i}$ and $b_{j}$ all being real or complex numbers. In this section, we show that this result remains valid if the real or complex field is replaced by any division subring of the quaternions.

Let $D$ be any division subring of the real quaternions $H$. Let $\oplus D$ be the direct sum of $D$, that is, the linear space, over $D$, of all finitely nonzero 
sequences from $D$. Put a form $($,$) on \oplus D$ by defining $(a, b)$ exactly as in the last paragraph, except that now the $a_{i}$ and $b_{j}$ are, of course, elements of $D$. We observe that $($,$) need not be a D$-valued form, since $D$ need not be closed under conjugation. For each subset $S$ of $\oplus D$, define $S^{\perp}=\{x \in \oplus D:(x, y)=0$ for each $y \in S\}$. Call a subspace $S$ of $\oplus D$ $\perp$-closed in case $S=S^{\perp \perp}$. It is easy to prove that the map $S \rightarrow S^{\perp \perp}$ is a closure operator on the lattice of all subspaces of $\oplus D$. Hence the set $L$ of all $\perp$-closed subspaces of $\oplus D$ is a complete, orthocomplemented lattice $[4$, p. 1518]. The main result of this paper is the following

\subsection{THEOREM. The lattice $L$ is nonorthomodular.}

The result of Kaplansky, referred to in the Introduction, from which we derive 3.1 , is the following:

3.2. TheOREM (KAPlanSKy). Let $D$ be a division ring with involution $k \rightarrow k^{*}$, let $V$ be a left vector space over $D$ of countably infinite Hamel dimension, and let $($,$) be a Hermitian, conjugate-bilinear form on V$ (which thus maps $V \times V$ into $D$ ), which has no isotropic vectors. Then, the lattice $L$ of all $\perp$-closed subspaces of $V$ is nonorthomodular.

As we said in the Introduction, 3.1 is a special case of 3.2 if the division ring $D$ is closed under quaternionic conjugation. But if $D$ fails to be conjugate closed, the form $($,$) assumes values lying outside D$ and thus fails to satisfy the hypothesis of 3.2. It is our goal to prove nonorthomodularity in this case as well. To this end, we indicate, in the next paragraph, a proof of Kaplansky's theorem. Since our goal is to derive 3.1 , the proof we outline will apply only to the case $D$ a division subring of $H$ with the canonical form on $\oplus D$, but we remark that the construction used here, with some modifications, applies to $\oplus D, D$ any division ring.

Changing our notation slightly from that of $\$ 2$, let $\left\{e_{0}, e_{1}, e_{2}, \cdots\right\}$ be the canonical Hamel basis for $\oplus D$, that is, $e_{0}=(1,0,0, \cdots), e_{1}=(0,1$, $0,0, \cdots)$, etc. Define a set of vectors $\left\{f_{0}, f_{1}, \cdots\right\}$ by the rule (for $n=0$, $1,2, \cdots) f_{n}=\sum\left(a_{i n} e_{i}: i=0,1, \cdots, n\right)$, with $a_{i n}$ defined, for each $n$, by

$$
\begin{aligned}
a_{i n}=1 & \text { if } i=0 \text { or } i=n, \\
=b_{i} & \text { if } 1 \leqq i<n,
\end{aligned}
$$

where the $b_{i}$ are defined inductively by

$$
\begin{aligned}
b_{i} & =-1 & & \text { if } i=1, \\
& =b_{i-1}-b_{i-1}^{2} & & \text { if } i=2,3, \cdots .
\end{aligned}
$$

Note that all $a_{i n}$ are rational so each $f_{i} \in \oplus D$. Also, for $i>0$, the vectors $f_{i}$ are pairwise orthogonal while $f_{0}$ (which equals $e_{0}$ ) fails to be orthogonal 
to any $f_{i}$. Letting $M$ be the subspace of $\oplus D$ spanned (algebraically) by $\left\{f_{1}, f_{3}, f_{5}, \cdots\right\}$ and $N$ the subspace spanned by $\left\{f_{2}, f_{4}, \cdots\right\}$, we can prove that $N=M^{\perp}$ and $M=N^{\perp}$, so that $M$ and $N$ are both $\perp$-closed subspaces of $\oplus D$. Finally, it is also true that $M \vee N=\oplus D$, but that $f_{0} \notin M+N$, so that $M+N$ is a non- $\perp$-closed subspace.

With this, we have shown that, for $D$ any division subring of the quaternions, there exists a pair of orthogonal $\perp$-closed subspaces of $\oplus D$ whose sum is not $\perp$-closed. In the case $D$ conjugate closed, and indeed in the more general setting of 3.2 (with the appropriate generalizations of the above construction having been carried out), this is enough to ensure the nonorthomodularity of $L$, because by Theorem III- 1 of [6], the closure operator $M \rightarrow M^{\perp \perp}$, mapping the lattice of all subspaces of $\oplus D$ into itself, is a Mackey closure operator [4, p. 1518]. Thus, by Theorem III-6 of [6], the existence of nonclosed orthogonal sums is equivalent to the existence of orthogonal, non-dual-modular pairs in the lattice, which is tantamount to nonorthomodularity [7, 29.13( $\gamma)]$. But, for the case $D$ non-conjugate-closed, the closure operator just mentioned is necessarily non-Mackey (as in $\S 2$, we can find nonclosed one-dimensional subspaces in $\oplus D$ ). Thus, we must turn to the problem of showing that the specific pair $M, N$ produced in the last paragraph is a non-dualmodular pair in $L$. This we do by proving two results, based on III- 1 and III-6 of [6], which depend on the specific construction of $M$ and $N$ for their validity.

\subsection{Lemma. The subspace $N+D e_{0}$ of $\oplus D$ is $\perp$-closed.}

PROOF. First note that, letting $y=f_{1}=e_{0}-e_{1}$, we have that $y \in N^{\perp}(=M)$ and $\left(y, e_{0}\right)=1$. Thus, for each $z \in N^{\perp},\left(z, e_{0}\right)-\left(z, e_{0}\right)\left(y, e_{0}\right)=\left(z, e_{0}\right)-$ $\left(z, e_{0}\right)=0$. Hence $\left(z-\left(z, e_{0}\right) y, e_{0}\right)=0$ for all $z \in N^{\perp}$ or, since $\left(z, e_{0}\right) \in D$, the vector $z-\left(z, e_{0}\right) y \in\left(D e_{0}\right)^{\perp}$ for all $z \in N^{\perp}$. Therefore, for any $z \in N^{\perp}$, $z-\left(z, e_{0}\right) y \in\left(N+D e_{0}\right)^{\perp}$. Now, let $w$ be any vector in $\left(N+D e_{0}\right)^{\perp \perp}$. Then, for any $z \in N^{\perp},\left(z-\left(z, e_{0}\right) y, w\right)=0$. This means that $(z, w)-\left(z, e_{0}\right)(y, w)=0$ for all $z \in N^{\perp}$, or $\left(z, w-(y, w)^{*} e_{0}\right)=0$ for all $z \in N^{\perp}$, or $w-(y, w)^{*} e_{0} \in$ $N^{\perp \perp}=N$. Since $(y, w)^{*}=(w, y) \in D$, by the original choice of $y$, we have that $w=v+(w, y) e_{0}$, for some $v \in N$, or $w \in N+D e_{0}$. Hence, $\left(N+D e_{0}\right)^{\perp \perp} \subseteq$ $N+D e_{0}$, as desired.

\subsection{Lemma. The pair $(M, N)$ is a non-dual-modular pair in $L$.}

Proof. To prove that $M$ and $N$ form a non-dual-modular pair, we must produce a $\perp$-closed subspace $K \geqq N$ such that $K \wedge(M \vee N)_{\nexists}^{\rightarrow}(K \wedge M) \vee$ $N$. Let $K=N+D e_{0} . K$ is $\perp$-closed by 3.3. Since $M \vee N=\oplus D$, then $K \wedge(M \vee N)=K$ so $e_{0} \in K \wedge(M \vee N)$. However $K \wedge M=0$. For if $m \in K \wedge M$, then $m=v+d e_{0}$ for some $v \in N$ and $d \in D$. If $d=0$, then $m=v \in N$ so 
$m \in M \wedge N=0$ so $m=0$. Thus $d \neq 0$. Then $e_{0}=d^{-1}(m-v)$ so that $e_{0} \in M+N$. But we have already seen that this is false (recall $e_{0}=f_{0}$ ). Hence $K \wedge M=0$ so that $(K \wedge M) \vee N=N$. Hence $e_{0} \notin(K \wedge M) \vee N$, so $K \wedge(M \vee N) \supsetneqq(K \wedge M) \vee N$.

With this, Theorem 3.1, our main result, is proved.

4. Concluding remark. We conclude by posing a question, whose affirmative answer would imply our main result and give more information about the class of lattices we are studying. The question is suggested by a definition due to D. E. Catlin [2]. An atomic, orthocomplemented lattice $L$ is said to have the $\sigma$-hyperoctant property if, for every countable orthogonal family of atoms $\left\{a_{i}: i=1,2, \cdots\right\}$ in $L$, there exists an atom $a \in L$ such that $a \leqq \bigvee\left\{a_{i}: i=1,2, \cdots\right\}$, but $a$ fails to commute with any of the $a_{i}$. Certainly, the lattices arising from the direct sums, discussed above, do not have this property. We ask whether an infinite-dimensional Hilbert lattice necessarily has the $\sigma$-hyperoctant property.

\section{REFERENCES}

1. I. Amemiya and H. Araki, A remark on Piron's paper, Publ. Res. Inst. Math. Sci. Ser. A 2 (1966/67), 423-427. MR 35 \#130.

2. D. E. Catlin, Cyclic atoms in orthomodular lattices, Proc. Amer. Math. Soc. 30 (1971), 412-418.

3. H. R. Fischer and H. Gross, Quadratic forms and linear topologies. I, Math. Ann. 157 (1964), 296-325. MR 30 \#3149.

4. S. S. Holland, Jr., Partial solution to Mackey's problem about modular pairs and completeness, Canad. J. Math. 21 (1969), 1518-1525. MR 40 \#6240.

5. I. Kaplansky, Forms in infinite-dimensional spaces, An. Acad. Brasil. Ci. 22 (1950), 1-17. MR 12, 238.

6. G. W. Mackey, On infinite dimensional linear spaces, Trans. Amer. Math. Soc. 57 (1945), 155-207. MR 6, 274; 7, 620.

7. F. Maeda and S. Maeda, Theory of symmetric lattices, Springer-Verlag, Berlin, 1970.

8. R. P. Morash, The orthomodular identity and metric completeness of the coordinatizing division ring, Proc. Amer. Math. Soc. 27 (1971), 446-448. MR 42 \#7570.

9. $\longrightarrow$, Erratum to [8], Proc. Amer. Math. Soc. 29 (1971), 627. MR 43 \#2016.

Department of Mathematics and Statistics, University of Massachusetts, AMHERST, MASSACHUSETTS 01002

Current address: Department of Mathematics, University of Michigan at Dearborn, Dearborn, Michigan 48128 